\title{
Recurrent breast carcinoma after prior intensive therapy responsive to biochemical modulation of 5-FU with low-dose cisplatin : Case report
}

\section{ORIGINAL ARTICLE Annals of Cancer Research and Therapy}

\author{
Shoji Oura, Takeo Sakurai, Goro Yoshimura, \\ Takeshi Tamaki, Teiji Umemura, Yozo Kokawa*
}

\begin{abstract}
A 53-year-old woman with breast carcinoma underwent modified radical mastectomy and intensive adjuvant chemoendocrine therapy (including CAF therapy), but developed bone metastasis. After resection of the metastatic focus and further chemoendocrine therapy, she developed multiple bone metastases and pain in the right upper arm. One course of 5 -fluorouracil combined with low-dose cisplatin achieved remarkable pain relief and new calcification of the lytic lesion in the humerus. There were no major side effects of this therapy. Unfortunately, spinal cord injury due to vertebral compression fracture forced us to discontinue treatment, so the duration of partial remission was less than two months. However, the response to this modulation regimen in a patient with very intensive prior therapy is notable.
\end{abstract}

Ann Cancer Res Ther 5(1) : 49 51, 1996/Received 22 May 1996, Accepted 21 Jun 1996 Key words : recurrent breast carcinoma, biochemical modulation, 5-FU, cisplatin

Anthracyclines (e. g. adriamycin, epirubicin and pirarubicin) are widely used for the treatment of advanced or recurrent breast carcinoma because of the high response rates obtained. However, anthracyclines also induce multidrug resistance $\left(\mathrm{MDR}^{1 \sim 4)}\right)$. Most oncologists treating breast cancer patients therefore encounter many difficulties after the administration of anthracyclines. Here we report a patient with recurrent breast carcinoma who had received intensive prior therapy including anthracyclines, but responded to biochemical modulation ${ }^{5,6)}$ of 5fluorouracil (5-FU) with low-dose cisplatin.

\section{Case report}

A 53-year-old woman with breast carcinoma underwent modified radical mastectomy in November 1989. As adjuvant therapy, she received five courses of CAF therapy (cyclophosphamide, adriamycin, and 5-FU) and one course of CMF therapy (cyclophosphamide, methotrexate, and 5-FU), followed by tamoxifen and oral 5-FU for three years. In January 1993, she suddenly developed left leg paresis. Radiological examination revealed bone metastasis in the 9th thoracic vertebra. On May 25, 1993, after one course each of CEF therapy (cyclophosphamide, epirubicin, and 5-FU) and CPP therapy (cyclophosphamide, pirarubicin, and cisplatin) combined with oral medroxyprogesterone acetate, we resected the bone metas-

\footnotetext{
* The Department of Surgery, Kihoku Hospital, Wakayama Medical College

Correspondence to: Shoji Oura, The Department of Surgery, Kihoku Hospital, Wakayama Medical College, 219 Myoji, Katsuragi-cho, Itogun, Wakayama 649-71, Japan. TEL0736-22-0066, FAX0736-22-2579
}

tasis. She obtained marked improvement of left leg function after this operation. However, she gradually developed pain in her right upper arm despite several types of chemoendocrine therapy (Fig. 1). Plain $x$-ray disclosed an osteolytic lesion in the right humerus (Fig. 2a), and hot spots were present in the vertebrae, ribs, and right humerus on a bone scintigram (Fig. 3). Given the findings described above, we judged that the hot areas were metastases. We therefore planned to treat the patient with additional chemotherapy. However, her prior intensive chemotherapy, including anthracyclines, made the choice of an appropriate regimen difficult. Conventional combination chemotherapy was likely to be ineffective, so we treated her using biochemical modulation of 5-FU with low-dose cisplatin. This modulation comprised infusion of cisplatin (10 mg in $100 \mathrm{ml}$ of saline) on days $1-5$ and 8-12 plus oral administration of $5-\mathrm{FU}(300 \mathrm{mg})$ on days 1-14. This regimen was repeated every four weeks.

The patient experienced remarkable pain relief and discontinued analgesics during the first course of chemotherapy. At the time of initiation of the second course, $X$-ray films of the affected humerus revealed new calcification (Fig. 2b). There were no major side effects of this therapy. One month after the appearance of new calcification, we judged her to have achieved partial remission (PR). Unfortunately, we were forced to discontinue therapy because spinal cord damage due to vertebral compression fracture led to her admission to the neurosurgical department of another hospital. As a result, the total duration of partial remission was less than two months On June 1, 1995, she died of meningeal carcinomatosis. 


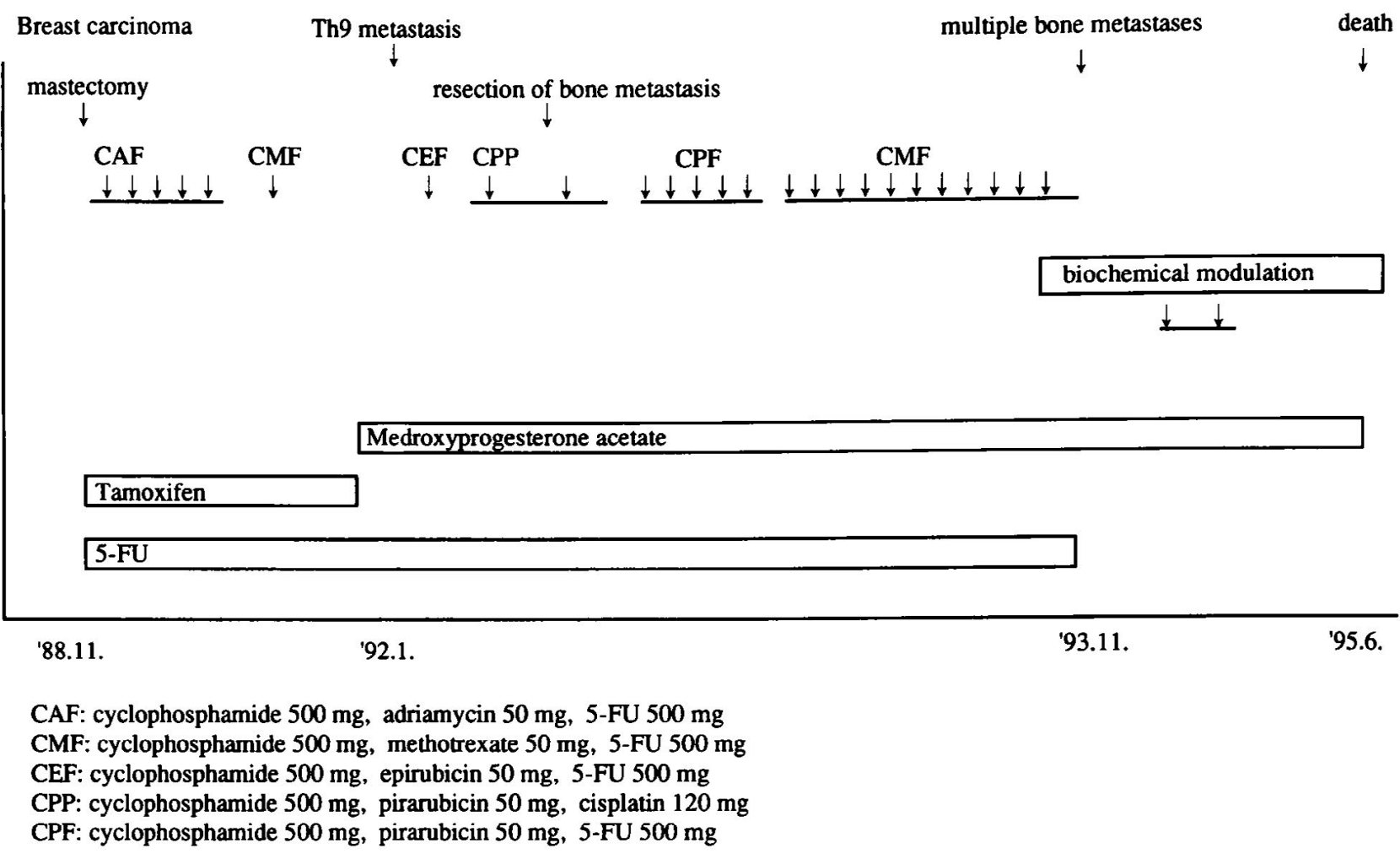

Fig. 1

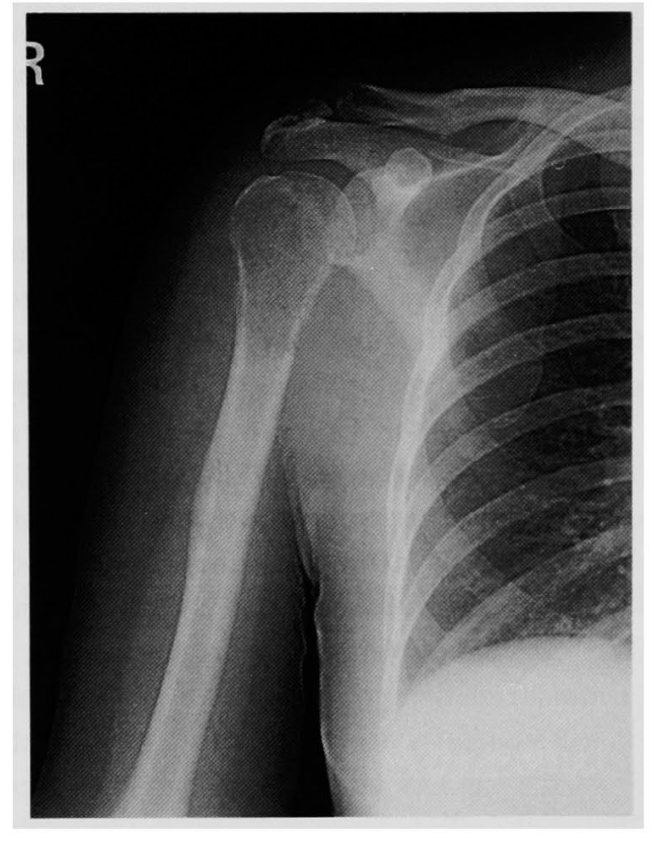

Fig. 2a

\section{Discussion}

The weakened antitumor activity of endocrine therapy or chemotherapy for recurrent breast carcinoma is primarily due to tumor heterogeniety and drug resistance result-

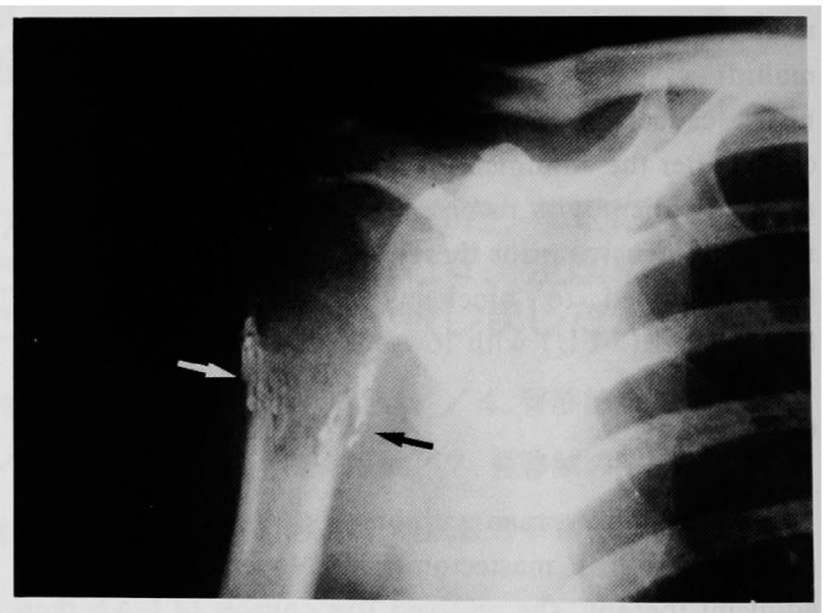

Fig. 2b

ing from prior therapy. Of these two factors, oncologists are usually more concerned with drug resistance. Multidrug resistance (MDR), which often develops following the use of anthracyclines, is a major problem in the treatment of recurrent breast carcinoma.

Some patients with local recurrence ${ }^{7,8)}$ or lung metastasis $^{9)}$ can be cured by surgery. However, patients with bone metastasis usually have a relatively long survival but are seldom cured. Therefore, most patients with bone metastasis have a history of intensive prior therapy at the terminal stage, and principally due to MDR, their disease 


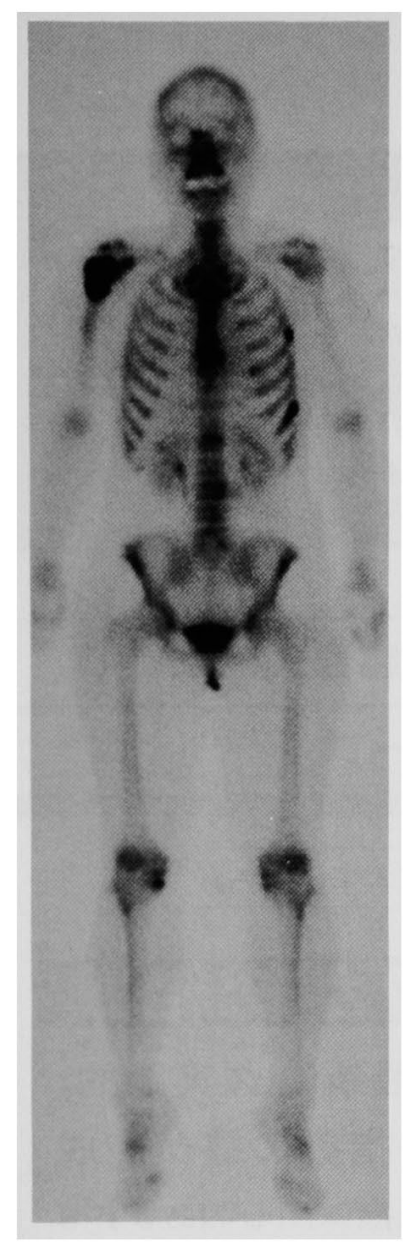

Fig. 3

cannot be controlled by any antitumor agents. As a result, progressive disease is usually treated by palliative radiotherapy and/or strong narcotics (e. g., morphine) for pain.

In the present patient, anthracyclines were given around the time of the primary operation and her spinal surgery but were not given just before the biochemical modulation therapy. Hence, it was unclear whether the patient had MDR or not at initiation of this therapy. However, we believe she had developed MDR, for the following reasons. First, she had already received three anthracyclines (adriamycin, epirubicin, and pirarubicin). Second, total dose of anthracyclines was $650 \mathrm{mg}$. Third, she had reeived diverse types of chemoendocrine therapy (includ- ing CMF therapy) in addition to anthracyclinecontaining chemotherapy (Fig. 1).

We could not precisely assess the usefulness of her biochemical modulation therapy, because we were forced to discontinue treatment after compression fracture led to admission to another hospital and made it impossible to observe her clinical course in detail. However, it was noteworthy that she obtained a response, even if transient, without serious side effects after biochemical modulation therapy, since she had already received intensive 5-FU therapy and high-dose cisplatin.

In conclusion, our findings suggest that breast oncologists should not only establish appropriate criteria for the administration of promising new antitumor agents such as taxol, tatotere, and CPT-11, but should also re-evaluate the usefulness of biochemical modulation employing conventional agents.

\section{References}

1) Tsuruo T. Mechanism of multidrug resistance and implications for therapy. Jpn J Cancer Res, 79: 285-296, 1988.

2) Fujimura T, Shibata H, Maekawa I, Furusawa S, Kawauchi H. Sasaki K, Takayanagi Y. Reversal of resistance to doxorubicin with cepharantine in murine P388 leukemia cells. Jpn J Pharmacol, 54 : 464-467, 1990.

3) Ishida $Y$, Shimada $Y$, Shimoyama M. Synergistic effect of cyclosporin A and verapamil in overcoming vincristine resistance of multidrug-resistant cultured human leukemia cells. Jpn J Cancer Res, 81 : 834-841, 1990.

4) Ishida $H$, Okabe $M$, Gomi $K$, Horiuchi R, Mikami K, Naito $M$, Tsuruo T. Modulation of adriamycin resistance in human breast carcinoma MCF-7 cells in vitro and in vivo by medroxyprogesterone acetate. Jpn J Cancer Res, 85 : 542-549, 1994.

5) Scanlon KJ, Newman EM, Lu Y, Priest DG. Biochemical basis for cisplatin and 5-fluorouracil synergism in human ovarian carcinoma cells. Proc Natl Acad Sci USA, 83: 8923-8925, 1986.

6) Shirasaka $T$, Shimamoto $Y$, Ohshima $H$. Saito $H$, Fukushima M. Metabolic basis of synergistic antitumor activities of 5 fluorouracil and cisplatin in rodent tumor models in vivo. Cancer Chemother Pharmacol, 32: 167-172, 1993.

7) Senoo $\mathbf{T}$ : Indications for surgical treatment of locally recurrent breast cancer. Jpn J Breast Cancer, 4: 51-59, 1989, (in Japanese)

8) Dahlstrom KK, Andersson AP, Andersen M, Krag C. Wide local excision of recurrent breast cancer in the thoracic wall. Cancer 72: 774-777, 1993

9) Nakagawa K, Tsuchiya S, Matsubara T, Tsuchiya E. Surgical treatment of pulmonary metastasis from breast cancer. Jpn $\mathrm{J}$ Breast Cancer, 3 : 179-187, 1988, (in Japanese). 